\title{
Canine babesiosis: a perspective on clinical complications, biomarkers, and treatment
}

This article was published in the following Dove Press journal:

Veterinary Medicine: Research and Reports

10 April 2015

Number of times this article has been viewed

\author{
Liza S Köster' \\ Remo G Lobetti² \\ Patrick Kelly' \\ 'Department of Clinical Sciences, \\ One Health Center for Zoonoses \\ and Tropical Veterinary Medicine, \\ Ross University School of Veterinary \\ Medicine, St Kitts, West Indies; \\ ${ }^{2}$ Bryanston Veterinary Hospital, \\ Bryanston, South Africa
}

\begin{abstract}
Canine babesiosis is a common tick transmitted disease of dogs worldwide. A number of Babesia sp. can infect dogs and the spectrum is increasing as molecular methods are developed to differentiate organisms. Clinical signs are generally attributed to hemolysis caused by the organisms in the erythrocytes but in some animals with some Babesia spp. there can be an immune mediated component to the anemia and/or a severe inflammatory reaction associated. This complicated form of canine babesiosis is associated with high morbidity and mortality. A variety of clinical markers has been investigated to enable clinicians to provide more accurate prognoses and adapt their treatments which vary according to the infecting species. In this review, we discuss the taxonomy, clinical signs, diagnostic imaging, clinical biomarkers, treatment, and prophylaxis of one of the most common and important diseases of dogs worldwide.
\end{abstract}

Keywords: babesiosis, vector-borne disease, dog

\section{Introduction}

Canine babesiosis occurs worldwide and results from infections with a variety of Babesia spp., tick-borne hemoprotozoa that were first described by Babes in cattle with hemolytic anemia in 1888. ${ }^{1,2}$ There are now over 100 Babesia spp. reported in vertebrate hosts ${ }^{3}$ and it is thought that potentially all vertebrates, including people, can be infected with Babesia, largely depending on their suitability as hosts for tick vectors. ${ }^{4}$ Infections in dogs can cause considerable morbidity and mortality with the clinical signs and pathogenesis of canine babesiosis paralleling falciparum malaria in people. ${ }^{5-7}$ In our review of canine babesiosis, we describe the current taxonomy of canine Babesia spp. and their geographic distribution, clinical manifestations, diagnostic imaging findings, clinical biomarkers, treatment, and prophylaxis.

Most data in the literature describing complications and biomarkers as prognosticators have been obtained from dogs infected with Babesia rossi. Therefore, in our review the clinical signs and complications we describe are those seen in $B$. rossi infections. Where data are from infections with other species of Babesia, this is specifically noted.

\section{Taxonomy and geographic distribution}

The Babesia genus belongs to the order Piroplasmida in the phylum Apicomplexa and can be seen as non-pigment forming pear or signet-ring shaped organisms in mammalian erythrocytes. ${ }^{4}$ They are mostly tick transmitted and are the second most common blood-borne parasites of mammals after the trypanosomes. ${ }^{8}$ Asexual reproduction occurs in canine erythrocytes while the sexual phase occurs in a variety of ixodid ticks, 
which can transmit the organism transovarially. In the dog, Babesia was first described at the end of the 19th century and there are now four well-recognized canine species, Babesia canis, Babesia vogeli, B. rossi and Babesia gibsoni, and a number of less well recognized isolates. Initially, Babesia was classified according to its morphology in erythrocytes with "large" and "small" forms being recognized as B. canis and B. gibsoni, respectively. Later, subspecies of $B$. canis were proposed because, although they were morphologically identical, they had different tick vectors, cross-immunity, and pathogenicity. With the advent of molecular phylogenetic analysis, in particular that of the $18 \mathrm{~S}$ rRNA gene, it was recognized that the subspecies are in fact distinct species, mainly B. rossi, B. canis, and B. vogeli. ${ }^{4,9,10}$ More recently an unnamed fourth "large" Babesia sp. (coco) has been found in dogs in North Carolina in the US. ${ }^{11}$ Genetic analyses have also shown there is a number of small Babesia spp. that are genotypically distinct from $B$. gibsoni. These include Babesia conradae and Babesia microti-like organisms, which are in separate lineages or clades. ${ }^{4,9}$

In their canine hosts, the Babesia spp. may cause hostmediated pathology and hemolysis with anemia, hyperbilirubinuria, hemoglobinuria, coagulopathies, and organ failure. Of the canine agents, $B$. rossi is endemic in southern Africa but has been reported elsewhere in Africa. ${ }^{12}$ It is transmitted by Haemaphysalis elliptica, previously Haemaphysalis leachi, and is the most virulent of the canine Babesia, generally causing marked hemolytic anemia that is often associated with complications (see "Clinical Signs"). ${ }^{13}$ B. canis is endemic in Europe but has been reported sporadically around the world. ${ }^{14}$ It is transmitted by Dermacentor spp. and generally only causes mild clinical signs which might include anorexia, depression, fever, jaundice, anemia, and thrombocytopenia. ${ }^{1}$ B. vogeli is found worldwide and is transmitted by Rhipicephalus sanguineus. ${ }^{15}$ It seldom causes clinical signs. Babesia sp. (coco) has only been found in immunosuppressed dogs where it has been associated with anemia and thrombocytopenia. ${ }^{11}$ Although the mode of transmission is unknown, ticks are suspected to be the vector.

B. gibsoni is the most prevalent of the "small" Babesia and is endemic in Asia where it is thought to be transmitted by Haemaphysalis longicornis. It also occurs sporadically in the rest of the world, probably because it can be transmitted by blood exchange when dogs fight. ${ }^{16}$ While infections may be subclinical, B. gibsoni is moderately pathogenic although complications are not common. B. conradae is considered more pathogenic and causes higher parasitemia, more pronounced anemias, and higher mortality (43\% versus
16\%). ${ }^{17}$ To date it has only been described in California and, although $R$. sanguineus is a possible vector, its method of transmission is unknown. ${ }^{16,17}$ The Babesia microti-like organism is endemic in northwest Spain and causes severe anemia and thrombocytopenia with azotemia also being common. ${ }^{18}$ The suspected vector is Ixodes hexagonus. ${ }^{19}$

\section{Clinical signs}

The clinical signs and outcome of canine babesiosis depends upon the infecting species, signalment, and host immunity. The incubation period is around 10-28 days which means the disease manifests after the vector tick has fed and detached from its host, a process which is usually complete within a week. ${ }^{20}$ Most infections are reported in spring and/or summer and are characterized by fever, lethargy, and varying degrees of hemolytic anemia with associated signs. Following the acute phase most dogs become chronically infected with no or only poorly characterized signs. To a large extent the signs shown and outcome of infection depend on the Babesia spp. involved. ${ }^{21-24}$

\section{Infections with $B$. vogeli}

This is the least virulent species and, although it can cause clinical disease due to severe anemia in puppies, it usually causes subclinical infections with a low parasitemia in adult dogs. The lack of virulence might be because of its long association with the domestic dog. ${ }^{23,25}$

\section{Infections with B. canis and B. rossi}

$B$. canis mostly causes mild signs while $B$. rossi is often associated with more sever disease. A highly virulent, peracute to acute form of canine babesiosis caused by B. rossi, occurs in South Africa and is also reported in Europe where it is due to $B$. canis $; 31 \%$ of affected dogs require hospital admission for intensive treatment. ${ }^{22,25,26}$ Dogs are usually presented with malaise and a history including, anorexia, lethargy, presence of ticks, and pigmenturia. ${ }^{21}$ Clinical abnormalities detected at presentation include fever, lethargy, weakness or collapse, pale mucous membranes, icterus, splenomegaly and, more rarely, lymphadenopathy and tremors..$^{20,21}$ The infection is characterized not only by hemolytic anemia (intravascular and extravascular) which is the hallmark manifestation of infections with Babesia spp., but also by a number of complications which are often fatal $(30 \%) .{ }^{5}$ The severity of anemia due to erythrocyte destruction varies from mild (hematocrit $[\mathrm{Ht}]<0.15-0.30 \mathrm{~L} / \mathrm{L})$ to severe $(\mathrm{Ht}<0.15 \mathrm{~L} / \mathrm{L}) .{ }^{27}$ Even in cases with low parasitemia, anemia can be profound, which suggests that non-parasite factors play a role, including 
peripheral sludging of capillaries, erythrophagocytosis by the spleen and liver and possibly immunoglobulin and complement-mediated destruction of erythrocytes. ${ }^{28}$

In some dogs, not all the clinical changes can be attributed to the hemolytic anemia and hypoxia; these dogs are regarded as having complicated canine babesiosis, a description loosely based on the World Health Organization (WHO) classification for malaria. ${ }^{5}$ In addition to the Babesia induced hemolysis, dogs with complicated babesiosis have immune mediated hemolytic anemia (IMHA) and/or signs from inflammatory reactions. ${ }^{29}$ Dogs with complicated forms of babesiosis are extremely challenging to treat with a mortality rate reportedly to be as high as $45 \%$ in one study. ${ }^{6}$ Further, there is a higher mortality rate $(29 \%)$ in dogs that develop a severe inflammatory reaction than in those that develop severe anemia ( $8 \%$ ), indicating the severity of the inflammatory response. ${ }^{29}$

Abnormalities seen in complicated canine babesiosis cases include hepatopathy, acute kidney injury (AKI), cerebral babesiosis, acute respiratory distress syndrome (ARDS), relative hemoconcentration ("red biliary"), pancreatitis and rhabdomyolysis, and myocardial dysfunction. 5,6,20,27,29-34

\section{Hepatopathy}

This is a complication of babesiosis evidenced by bilirubinemia, pigmenturia, and icterus, a sign seldom seen in dogs with only hemolysis. ${ }^{5,34}$ Hepatopathies commonly occur with other complications, particularly pancreatitis, and are not associated with a poor outcome when they occur as the only complication. Dogs with hepatopathies, however, may have delayed recoveries. ${ }^{5}$

\section{AKI}

$\mathrm{AKI}$ is an uncommon complication of babesiosis and typically presents as anuria or oliguria despite adequate hydration. At necropsy, the kidneys are swollen and dark in color, with redbrown urine in the bladder. Microscopically the renal tubular epithelial (RTE) cells are swollen and contain hemoglobin droplets and small vacuoles. ${ }^{34}$ Necrosis can be evident in severe cases. Multiple hemoglobin casts are also often present in the nephron lumen. ${ }^{28}$ These morphologic lesions have been attributed to anemic hypoxia resulting from erythrocyte destruction. However, in cases of complicated babesiosis with elevated creatinine the mean $\mathrm{Ht}$ is significantly higher, making hypovolemia a more likely cause than anemia for renal insufficiency in canine babesiosis. ${ }^{31}$

Renal changes in babesiosis have also been attributed to hemoglobinuria and referred to as hemoglobinuric nephropathy.
One study, however, showed that hemoglobinuria, of the magnitude seen in canine babesiosis, did not induce a significant nephropathy, regardless of the presence of concomitant anemia. The study also showed that the glomerular filtration rate (GFR) was reduced in dogs that were rendered as anemic as dogs with severe babesiosis and this might then be one mechanism of kidney injury. ${ }^{35}$ In another study, dogs with naturally occurring babesiosis had significant urine met-hemoglobin with no evidence of blood met-hemoglobin implying that the urinary met-hemoglobin was either produced in the kidney or possibly by oxidation of hemoglobin to met-hemoglobin in the bladder. ${ }^{36}$ Met-hemoglobin has been shown experimentally to be toxic and might then be another cause of renal injury in canine babesiosis.

Proteinuria, RTE celluria, variable enzymuria, and azotemia have been demonstrated in dogs with babesiosis but these are all minimal changes and consistent with hypoxia, reduced GFR, or reduced cardiac output. ${ }^{31}$ Urinalysis typically shows hypersthenuria, bilirubinuria, hemoglobinuria, proteinuria, granular casts, and RTE cells.

\section{Cerebral babesiosis}

Occurring uncommonly, cerebral babesiosis carries a poor prognosis and is caused by endothelial damage with subsequent microvascular necrosis, perivascular edema, and hemorrhage. ${ }^{27}$ The disease has a hyperacute onset and the pathology is characterized by "sludging" of parasitized erythrocytes in the small vessels of the brain. ${ }^{5}$ The rate of progression is rapid with a dog reported to become tetraplegic within hours of presenting with hind limb ataxia; most dogs will show neurological signs including seizures, behavioral changes, ataxia, paresis, nystagmus, anisocoria, and paddling with vocalization. ${ }^{5,34}$ Both acute and delayed onset cerebellar ataxia, have also been described dogs with babesiosis. ${ }^{37}$

\section{ARDS}

This syndrome is defined by an arterial partial pressure $\left(\mathrm{PaO}_{2}\right)<60 \mathrm{mmHg}$, an alveolar to arterial oxygen gradient $>15 \mathrm{mmHg}$ (inspired oxygen $\left[\mathrm{FiO}_{2}\right]$ of room air) and is clinically characterized dyspnea with agonal blood-tinged pulmonary edema from the nostrils. In dogs with canine babesiosis it has been reported as "shock lung" and is responsible for the highest numbers of fatalities in complicated babesiosis. .,6 $^{5}$

\section{"Red biliary"}

This is a syndrome of paradoxical hemoconcentration with intravascular hemolysis and is a rare complication 
in canine babesiosis. The cause is thought to be vasculitis and fluid shifts leading to relative hemoconcentration ( $\mathrm{Ht}>0.47 \mathrm{~L} / \mathrm{L}$ ) despite severe hemolysis. ${ }^{6}$ Red biliary is often associated with both AKI and cerebral babesiosis and has a guarded prognosis. ${ }^{5,6}$

\section{Rhabdomyolysis}

Rhabdomyolysis is characterized clinically by muscle pain, tremors, and pigmenturia. ${ }^{34,38}$ It is seen rarely and can be accompanied by other complications including AKI, cerebral babesiosis, and ARDS. There is increased serum myoglobin, muscle enzymes, muscle necrosis, and hemorrhage on necropsy. ${ }^{38}$

\section{Pancreatitis}

This was previously referred to as the "gut form" of babesiosis and is associated with gastrointestinal clinical signs including anorexia, vomiting, diarrhea, melena, hematemesis, and abdominal effusion. ${ }^{32}$ The mortality rate is reported to be $21 \%$ with other complications occurring frequently in dogs with acute pancreatitis: clinical icterus (65\%), IMHA $(30 \%)$, AKI (15\%), cerebral babesiosis $(10 \%)$, and hemoconcentration $(10 \%)$.

\section{Cardiac dysfunction}

Cardiac dysfunction in canine babesiosis has traditionally been regarded as a rare complication, with the majority of lesions reported as incidental findings at post-mortem examination. Macroscopic cardiac lesions that have been reported include pericardial effusion and pericardial, epicardial, and endocardial hemorrhage, which usually involve one or more of the chambers with the left ventricle being most commonly affected. Documented cardiac histopathology changes are hemorrhage, necrosis, inflammation, and fibrin micro-thrombi in the myocardium. Lesions may be multifocal but more generally they are limited to one area within the myocardium. ${ }^{39}$ With cardiac dysfunction there is reduced renal blood flow and glomerular filtration due to redistribution of blood flow which is common in early heart failure. ${ }^{31}$

\section{Infections with $B$. gibsoni}

Most infections cause mild signs in the acute phase but some B. gibsoni infections can cause severe anemia and be misdiagnosed as IMHA either due to the parasite not being visible on blood films due to low parasitemia or due to low level of suspicion in non-endemic areas. ${ }^{40}$ Most dogs are depressed, have a history of anorexia, and are diagnosed with regenerative anemia. ${ }^{40,41}$ Many dogs are Coombs' test positive. Despite the thrombocytopenia, coagulation profiles are normal with no clinical signs of hemorrhage. ${ }^{42}$ A case of protein-losing nephropathy (membranoproliferative glomerulonephritis and immune complex deposition) has been described in a Labrador Retriever that was polymerase chain reaction positive for $B$. gibsoni and resolved with elimination of the infection. ${ }^{43}$ Cutaneous vasculitis secondary to B. gibsoni infection in a Satsuma dog was characterized by generalized alopecia, ear tip papules and erosions, and necrosis of the skin of the forelimb. The skin changes were due to immune complex adherence to blood vessel wall and perivascular pathology. ${ }^{44}$

Signs in chronic infections are not uncommon and include low-grade fever, pallor, splenomegaly, and lymphadenomegaly.

\section{Diagnostic imaging}

Hepatomegaly and splenomegaly are the most consistent findings on abdominal ultrasonography in dogs with B. canis. $^{21,45}$ The largest study $(\mathrm{n}=72)$ to describe ultrasonographic changes in dogs naturally infected with babesiosis, assumed to be $B$. canis, consistently found splenomegaly with diffuse, hypoechoic, heterogeneity. ${ }^{45}$ Despite only mild changes in creatinine and urea concentrations, renal changes have been noted in severe uncomplicated and complicated cases of babesiosis. ${ }^{21,45}$ Diffuse homogenous increased cortical echogenicity and increased corticomedullary definition have been attributed to the effects of hemoglobinuria, hypoxic damage, or immune-complex glomerulonephritis. Doppler characteristics were compared between dogs with babesiosis caused by B. rossi and dogs with severe anemia, the renal interlobar artery resistivity index (RI) was significantly higher in the former group due to reduced diastolic velocities. ${ }^{46}$ That study eloquently demonstrated that the differences in the Doppler characteristics between the two groups were due to the increased RI in the group of dogs with babesiosis and thus not solely due to anemia. In dogs infected with $B$. canis, the pulsatility index was significantly higher in dogs with severe uncomplicated babesiosis and RI was significantly higher in dogs with severe uncomplicated and complicated babesiosis. ${ }^{45,46}$ The increases in pulsatility index and RI could have been due to endothelin induced vasoconstriction and part of the initiation phase of AKI. It was found in $75 \%$ of dogs with renal involvement and $80 \%$ of dogs that died from AKI.

Ultrasonographic changes have been described in the pancreases of dogs infected with both B. rossi and B. canis that showed gastrointestinal signs and abdominal pain. ${ }^{21,32}$ 
These changes were consistent with acute pancreatitis and included duodenal atony and peripancreatic fat hyperechogenicity.

Pulmonary perfusion studies using scintigraphy to evaluate the possibility of pulmonary thromboembolism in canine babesiosis failed to show pleural-based pulmonary wedge shaped defects consistent with the condition. ${ }^{47}$

\section{Clinical biomarkers}

There is a variety of biomarkers, which might be helpful in determining the severity and outcome in dogs with canine babesiosis.

\section{Blood cell parameters}

Hematological changes have been examined in several investigations. ${ }^{21,48,49,50}$ Following infection there is usually a mild to moderately regenerative normocytic and normochromic anemia because of the hemolysis. ${ }^{51}$ Hemoconcentration indicates a poor prognosis, as dogs with an elevated $\mathrm{Ht}$, or an $\mathrm{Ht}$ within reference range but inappropriate for the degree of hemolysis evident, can have very high mortality rates. ${ }^{29}$ Neutrophil counts are usually normal to decreased although left shifts are relatively common. Thrombocytopenia is a consistent finding and is usually severe in the acute phase of infection. Platelet counts usually rise within a week of therapy. The severity and rapid recovery of the platelet counts has led to the suggestion that immune mediated mechanisms are involved.

\section{Coagulation profiles}

Coagulopathies, both primary and secondary hemostasis abnormalities, have been described in both $B$. canis and B. rossi infections. ${ }^{52-54}$ Thrombin-antithrombin complexes were found to be significantly higher and antithrombin activity significantly lower in $B$. canis infected dogs than controls..$^{53}$ Goddard et al demonstrated significant consumptive coagulopathy in non-survivors compared to survivors in dogs with B. rossi infection. ${ }^{52}$ Specifically, the median coagulation factor concentrations and protein $\mathrm{C}$ activity were lower in non-survivors and median $\mathrm{D}$-dimer concentration was higher in the dogs that died. In uncomplicated B. rossi infections, a transient coagulopathy with abnormalities in prothrombin time, activated partial thromboplastin time, fibrinogen, D-dimer, and thromboelastography was found in dogs despite lack of clinical hemorrhage. ${ }^{55}$ Infected dogs had significantly lower mean platelet count, higher fibrinogen concentrations, higher D-dimer value, prolonged activated partial thromboplastin time, and thromboelastography lysis values consistent with clots more resilient to lysis, as compared to healthy controls. Increased concentrations of intercellular adhesion molecule, CRP, and fibrinogen and decreased concentration of VWF were found in dogs in the acute stages of $B$. canis infection. ${ }^{54}$ The mechanism of the hemostatic dysfunction seen in canine babesiosis appears likely to be as a result of endothelial dysfunction and most likely contributes to red blood cell and thus parasite sequestration which may be an important factor in clinical outcome.

\section{Cytokines}

In canine babesiosis there is an association between TNF concentration, clinical severity as well as parasitemia. ${ }^{56} \mathrm{TNF}$ probably plays a role in the production of other harmful substances such as nitric oxide or free oxygen radicals and could mediate the interaction between parasitized erythrocytes and the blood vessel wall. Nitric oxide metabolites, nitrate and nitrite, collectively referred to as reactive nitrogen intermediates are proposed to be one of the mediators of a multiple organ dysfunction syndrome which is seen in complicated canine babesiosis, a putative sequela of systemic inflammatory response syndrome. ${ }^{57}$ In the acute virulent form of canine babesiosis, $87 \%$ of complicated cases fulfilled the requirements for SIRS. ${ }^{6}$ However, the concentrations of reactive nitrogen intermediates have not been shown to correlate significantly with the severity of illness nor is it predictive of outcome.

The concentration of AGP, a positive acute phase protein, is considerably elevated in dogs infected with $B$. rossi but levels do not correlate with severity of disease or outcome. ${ }^{58}$ The acute phase response, as measured by SAA and CRP concentrations, significantly decreases after successful treatment of $B$. canis infections, and together with platelet counts have been found to be the most sensitive parameters for monitoring therapeutic responses in infected dogs. ${ }^{59}$

A study of B. rossi infections has shown no differences in CRP levels between survivors and non-survivors. ${ }^{60}$ However, when included in a regression model together with glucose, CRP was significantly associated with outcome. In dogs with B. gibsoni, CRP has an inverse relationship with packed cell volume recovery and so could be a clinical marker of disease clearance. ${ }^{61}$ This supports the hypothesis that, although inflammatory mechanisms are important in the pathogenesis of babesiosis, tissue hypoxia and metabolic dysfunction play a major role in the disease process and outcome.

Statistically significant differences in the levels of MCP-1 have been found between control dogs $(118 \mathrm{pg} / \mathrm{mL})$ and 
Babesia-infected survivors (431 pg/mL) and non-survivors $(757 \mathrm{pg} / \mathrm{mL}){ }^{62}$

\section{Acid-base values}

Mixed metabolic and respiratory acid-base imbalances occur in canine babesiosis; arterial pH can vary from severe acidemia to alkalemia and there can be inappropriate hypocapnia with partial pressures of $\mathrm{CO}_{2}<10 \mathrm{mmHg}$ and negative base excesses due to unmeasured strong ions and hypoalbuminemic alkalosis. ${ }^{63}$ In a study of 34 dogs with the severe form of B. rossi infection, most dogs had concurrent metabolic acidosis and respiratory alkalosis, were hyperlactatemic, and had wide anion gaps, either high or low. ${ }^{63}$ Dogs with normal anion gaps often had concurrent hypoalbuminemia, which is common in this disease.

\section{Metabolic abnormalities}

Hypoglycemia is a common complication of canine babesiosis and is associated with collapse, severe anemia, age ( $<6$ months), and icterus. ${ }^{64}$ Reduced survival has reportedly been associated with hypoglycemia $(<59.4 \mathrm{mg} / \mathrm{dL})$ and hyperlactatemia (see below) at admission. ${ }^{65}$ Babesia infected dogs with hypoglycemia have very high TNF values, which correlate with parasitemia. ${ }^{56}$ Central nervous system signs resulting from low glucose levels may have been previously misdiagnosed as cerebral babesiosis.

Lactate has been established as a prognosticator in that mean lactate in non-survivors $(145 \mathrm{mg} / \mathrm{dL})$ was higher than in survivors $(13.8 \mathrm{mg} / \mathrm{dL})$. Pre-treatment hyperlactatemia $(>45 \mathrm{mg} / \mathrm{dL})$ and subsequent serial lactate concentrations that failed to return to normal reference range (persistently $>40 \mathrm{mg} / \mathrm{dL}$ ) indicated a poor prognosis. ${ }^{65}$

\section{Endocrine predictors}

Mortality is significantly associated with high cortisol and high adrenocorticotrophic hormone concentrations and low thyroxine and plasma free thyroxine concentrations. ${ }^{66}$

\section{Renal parameters}

Elevated serum urea levels are not uncommon in dogs with babesiosis and this elevation is often disproportionate to the rise in creatinine. This is probably due to increased protein catabolism and urea production resulting from gastrointestinal hemorrhage or protein catabolism due to the febrile inflammatory illness. ${ }^{31,67}$ In complicated babesiosis the serum urea and creatinine levels can be proportionately increased indicating decreased renal perfusion, possibly as a result of decreased blood pressure, decreased myocardial function, and/or hypovolemia. One study showed that elevated creatinine was associated with increased risk of death but it was not clear if the elevation was due to pre-renal azotemia. ${ }^{68} \mathrm{CST} 3$ has been proposed as a more sensitive marker than creatinine for reduced GFR due to renal insufficiency. However in one study both the mean and median serum CST3 concentrations of dogs with babesiosis were within reference range. ${ }^{69}$ It has been shown that dogs with $B$. rossi infections had significantly higher concentrations of urinary IgG, urinary CRP, and urinary RBP indicative of the presence of both glomerular and tubular dysfunction. ${ }^{30}$ These markers appear to be superior to specific gravity, and serum urea and creatinine concentrations for the early detection of renal dysfunction in dogs with babesiosis. Although urine enzyme (GGT and ALP) activity is both an early and persistent indicator of renal tubular damage, in canine babesiosis changes in urine enzyme activity appear to be variable and minimal, thus limiting their utility as diagnostic tests. ${ }^{31}$

\section{Central nervous system signs}

Central nervous system involvement results in a 57 times greater chance of death. ${ }^{6}$

\section{Respiratory signs}

Pulmonary edema is associated with the highest proportion of fatalities. ${ }^{6}$ This most likely is indicative of ARDS.

\section{Liver markers}

Icterus is reported to occur in advanced stages of canine babesiosis $^{34}$ with bilirubin values of over $170 \mu \mathrm{mol} / \mathrm{L}$ correlating positively with mortality.

High ALT activity is associated with a high risk of mortality. ${ }^{29}$

\section{Hemodynamic and cardiovascular changes}

Dogs with severe and complicated babesiosis are frequently presented in a state of collapse and clinical shock, with the shock resembling the hyperdynamic phase of acute septic shock. It is likely that hypotension in babesiosis results from a combination of vasodilation, reduced vascular volume due to increased vascular permeability and/or dehydration, and myocardial depression. ${ }^{70}$ In $B$. rossi infections, although hypotension correlates positively with disease severity and negatively with white cell counts, arterial blood pressure has not been shown to be significantly associated with survival. ${ }^{71}$ In the case of $B$. canis infections, no association was found between disease severity and arterial blood pressure. ${ }^{45}$ Other studies on dogs with $B$. canis infections, however, have 
shown that animals with septic shock, SIRS or multiple organ dysfunction syndrome with unresponsive hypotension had $100 \%$ mortality ${ }^{26} \mathrm{~A}$ study investigating circulatory collapse, parasitemia, and outcome in dogs infected with B. rossi showed that dogs that died had significantly higher capillary and venous parasitemia than dogs that survived and a high association between circulatory score and outcome. ${ }^{72}$

In dogs with babesiosis, increases in NT-proBNP have been documented with the elevations being proportional to the severity of the disease. This implies that dogs with babesiosis have a reduced cardiac function and that the dysfunction becomes more severe as the disease severity increases. ${ }^{73}$ The sensitive marker for myocardial injury, cardiac troponin I (cTnI), has also been shown to be increased in babesiosis with the magnitude of the elevation appearing to be directly proportional to the severity of the disease..$^{74}$ Dogs dying of canine babesiosis with histological evidence of myocardial lesions showed higher concentrations of $\mathrm{cTnI}$ than dogs that survived. cTns and NT-proBNP thus seem to be sensitive markers of myocardial injury in babesiosis and also may be useful in assessing the severity of the myocardial dysfunction.

A variety of arrhythmias have been reported in dogs with babesiosis including sino-atrial block, sinus arrest, first and second-degree atrioventricular block, ventricular premature depolarizations, and ventricular tachycardia. Other electrocardiogram (ECG) changes include low amplitude $\mathrm{R}$ waves, prolonged QRS interval, ST segment deviation, large T waves, and notching of the $\mathrm{R}$ wave. ${ }^{39}$ Although bradycardia and an irregular rhythm appear to be a poor prognostic indicator, ${ }^{39}$ in general the ECG abnormalities do not appear to be related to disease severity or to pathological changes and thus the clinical value of routine ECG monitoring in canine babesiosis seems limited. The only exception to this is when ventricular depolarizations and/or ventricular tachycardia is observed. These appear to be associated with elevated cTnI concentrations ${ }^{74}$ and although apparently specific for myocardial damage they are not always seen with myocardial damage. Although notching of the $\mathrm{R}$ wave can be indicative of myocardial infarction, in babesiosis notching of the $\mathrm{R}$ wave appears to be an inconsistent finding that is more commonly seen in dogs with secondary IMHA..$^{39,75}$

\section{Treatment}

Treatment for canine babesiosis consists of three components: treatment to eliminate the parasite, blood transfusions to treat severe anemia, and supportive care for the complications and metabolic derangements.
B. canis, B. rossi, and B. vogeli are most successfully treated with diminazene aceturate $(3.5 \mathrm{mg} / \mathrm{kg}$ subcutaneously or intramuscularly) or imidocarb dipropionate $(7.5 \mathrm{mg} / \mathrm{kg}$ once or $7 \mathrm{mg} / \mathrm{kg}$ twice, 14 days apart, intramuscularly). ${ }^{20}$ Administration of imidocarb dipropionate is associated with pain at the injection site and cholinergic signs, mainly salivation, vomiting, and diarrhea. With overdosing there can be massive liver necrosis. ${ }^{76}$ Diminazene can cause central nervous system toxicity in dogs, which appears to be dose related and can also occur with repeated administrations due to the drug's prolonged elimination half-life. ${ }^{77}$ Postmortem findings include bilateral cortical hemorrhages and edema which mimic lesions associated with cerebral babesiosis.

In the case of $B$. gibsoni, diminazene aceturate often fails to eliminate parasites. Attempts to sterilize infections using triple antibiotic combinations: doxycycline (5 mg/kg, orally, twice daily), clindamycin $(25 \mathrm{mg} / \mathrm{kg}$, orally, twice daily), metronidazole $(15 \mathrm{mg} / \mathrm{kg}$, orally, twice daily); or doxycycline (7-10 mg/kg, orally, twice daily), enrofloxacin (2-2.5 $\mathrm{mg} / \mathrm{kg}$, orally, twice daily), metronidazole $(5-15 \mathrm{mg} / \mathrm{kg}$, orally, twice daily) in combination with (6 weeks of oral antibiotics) and without (12 weeks of oral antibiotics) diminazene aceturate did not necessarily eliminate infections. ${ }^{61,78}$ Amphotericin B has exhibited activity against $B$. gibsoni but in vitro caused oxidative red blood cell damage and in vivo caused adverse kidney infection without elimination of the organism. ${ }^{79}$ Atovaquone (13.3 $\mathrm{mg} / \mathrm{kg}$, three times daily, orally for 10 days) in combination with azithromycin $(10 \mathrm{mg} / \mathrm{kg}$, once daily, orally for 10 days $)$ was the first described treatment combination to eliminate or suppress parasite numbers below detectable levels in the majority of dogs with no adverse reactions.$^{80}$ However, atovaquone alone ( $30 \mathrm{mg} / \mathrm{kg}$, twice daily, orally for 7 days) resulted in relapses and resistance ${ }^{81}$ and atovaquone $(17-25 \mathrm{mg} / \mathrm{kg})$ with proguanil $(7-10 \mathrm{mg} / \mathrm{kg}$ ) both twice daily for 10 days are effective in treating acute infections but do not eliminate $B$. gibsoni, even when combined with doxycycline. ${ }^{82}$

Supportive therapies and management of specific complications of canine babesiosis are not covered in this review as the reader is referred to reviews that are more specific. ${ }^{20,83}$

\section{Prophylaxis}

The primary means of prevention is tick control. Regular examination of dogs to remove ticks soon after they attach is important as it takes a minimum of 48 hours before Babesia transmission occurs. A large number of topical products are currently available for the control of ticks on dogs. ${ }^{84}$ The use of amitraz-impregnated collars has been 
shown to significantly reduce new infections in $B$. rossi endemic areas. ${ }^{85}$

Babesia can also be transmitted in whole blood and blood donors should be negative for babesiosis, preferably by polymerase chain reaction. It is also generally accepted that $B$. gibsoni can be transmitted by transfer of blood during dog fighting, which should be prevented.

The duration of protective immunity against canine babesiosis is limited with antibody titres declining approximately 5 months after infection. ${ }^{86}$ Seropositivity in itself does not guarantee protective immunity and cross protection between strains and species does not occur. ${ }^{86}$ In Europe a vaccine is available against $B$. canis with a reported efficacy of $70 \%-100 \% .{ }^{87}$ More recently a bivalent vaccine derived from soluble parasite antigens from $B$. canis and $B$. rossi has been shown to reduce duration and severity of clinical signs. ${ }^{88}$ Although vaccination against canine babesiosis does not prevent infection, it does seem to block the initiation of pathologic processes involved in the pathogenesis of the disease. ${ }^{89}$

\section{Disclosure}

The authors have no conflicts of interest in this work.

\section{References}

1. Boozer AL, Macintire DK. Canine babesiosis. Vet Clin North Am Small Anim Pract. 2003;33(4):885-904, viii.

2. Babes V. Sur l'hemoglobinurie bacterienne du boeuf [Bacterial hemoglobinuria in cattle]. Rend Acad Sci Ser Ser III Vie. 1888;107:692-694. French.

3. El-Bahnasawy MM, Khalil HH, Morsy TA. Babesiosis in an Egyptian boy acquired from pet dog, and a general review. J Egypt Soc Parasitol. 2011;41(1):99-108.

4. Schnittger L, RodriguezAE, Florin-Christensen M, Morrison DA. Babesia: a world emerging. Infect Genet Evol. 2012;12(8):1788-1809.

5. Jacobson LS, Clark IA. The pathophysiology of canine babesiosis: new approaches to an old puzzle. J S Afr Vet Assoc. 1994;65(3):134-145.

6. Welzl C, Leisewitz AL, Jacobson LS, Vaughan-Scott T, Myburgh E. Systemic inflammatory response syndrome and multiple-organ damage/ dysfunction in complicated canine babesiosis. J S Afr Vet Assoc. 2001; 72(3):158-162.

7. Clark IA, Jacobson LS. Do babesiosis and malaria share a common disease process? Ann Trop Med Parasitol. 1998;92(4):483-488.

8. Telford SR 3rd, Spielman A. Reservoir competence of white-footed mice for Babesia microti. J Med Entomol. 1993;30(1):223-227.

9. Lack JB, Reichard MV, Van Den Bussche RA. Phylogeny and evolution of the Piroplasmida as inferred from 18S rRNA sequences. Int $J$ Parasitol. 2012;42(4):353-363.

10. Uilenberg G. Babesia - a historical overview. Vet Parasitol. 2006; 138(1-2):3-10.

11. Marks Stowe DA, Birkenheuer AJ, Grindem CB. Pathology in practice. Intraerythrocytic infection with organisms consistent with a large Babesia sp. J Am Vet Med Assoc. 2012;241(8):1029-1031.

12. Oyamada M, Davoust B, Boni M, et al. Detection of Babesia canis rossi, B. canis vogeli, and Hepatozoon canis in dogs in a village of eastern Sudan by using a screening PCR and sequencing methodologies. Clin Diagn Lab Immunol. 2005;12(11):1343-1346.
13. Apanaskevich DA, Horak IG, Camicas JL. Redescription of Haemaphysalis (Rhipistoma) elliptica (Koch, 1844), an old taxon of the Haemaphysalis (Rhipistoma) leachi group from East and southern Africa, and of Haemaphysalis (Rhipistoma) leachi (Audouin, 1826) (Ixodida, Ixodidae). Onderstepoort J Vet Res. 2007;74(3):181-208.

14. Solano-Gallego L, Baneth G. Babesiosis in dogs and cats - expanding parasitological and clinical spectra. Vet Parasitol. 2011;181(1):48-60.

15. Inokuma $\mathrm{H}$, Yoshizaki $\mathrm{Y}$, Matsumoto $\mathrm{K}$, et al. Molecular survey of Babesia infection in dogs in Okinawa, Japan. Vet Parasitol. 2004; 121(3-4):341-346.

16. Irwin PJ. Canine babesiosis: from molecular taxonomy to control. Parasit Vectors. 2009;2 Suppl 1:S4.

17. Kjemtrup AM, Wainwright K, Miller M, Penzhorn BL, Carreno RA. Babesia conradae, sp. Nov., a small canine Babesia identified in California. Vet Parasitol. 2006;138(1-2):103-111.

18. Garcia AT. Piroplasma infection in dogs in northern Spain. Vet Parasitol. 2006;138(1-2):97-102.

19. Camacho AT, Pallas E, Gestal JJ, et al. Ixodes hexagonus is the main candidate as vector of Theileria annae in northwest Spain. Vet Parasitol. 2003;112(1-2):157-163.

20. Schoeman JP. Canine babesiosis. Onderstepoort J Vet Res. 2009; 76(1):59-66.

21. Mathe A, Voros K, Papp L, Reiczigel J. Clinical manifestations of canine babesiosis in Hungary (63 cases). Acta Vet Hung. 2006;54(3): 367-385.

22. Shakespeare AS. The incidence of canine babesiosis amongst sick dogs presented to the Onderstepoort Veterinary Academic Hospital. J S Afr Vet Assoc. 1995;66(4):247-250.

23. Irwin PJ, Hutchinson GW. Clinical and pathological findings of Babesia infection in dogs. Aust Vet J. 1991;68(6):204-209.

24. Collett MG. Survey of canine babesiosis in South Africa. J S Afr Vet Assoc. 2000;71(3):180-186.

25. Penzhorn BL. Why is Southern African canine babesiosis so virulent? An evolutionary perspective. Parasit Vectors. 2011;4:51.

26. Matijatko V, Kis I, Torti M, et al. Septic shock in canine babesiosis. Vet Parasitol. 2009;162(3-4):263-270.

27. Jacobson LS. The South African form of severe and complicated canine babesiosis: clinical advances 1994-2004. Vet Parasitol. 2006;138(1-2): 126-139.

28. Maegraith B, Gilles HM, Devakul K. Pathological processes in Babesia canis infections. Z Tropenmed Parasitol. 1957;8(4):485-514.

29. Reyers F, Leisewitz AL, Lobetti RG, et al. Canine babesiosis in South Africa: more than one disease. Does this serve as a model for falciparum malaria? Ann Trop Med Parasitol. 1998;92(4):503-511.

30. Defauw P, Schoeman JP, Smets P, et al. Assessment of renal dysfunction using urinary markers in canine babesiosis caused by Babesia rossi. Vet Parasitol. 2012;190(3-4):326-332.

31. Lobetti RG, Jacobson LS. Renal involvement in dogs with babesiosis. J S Afr Vet Assoc. 2001;72(1):23-28.

32. Mohr AJ, Lobetti RG, van der Lugt JJ. Acute pancreatitis: a newly recognised potential complication of canine babesiosis. J S Afr Vet Assoc. 2000;71(4):232-239.

33. Lobetti R. Changes in the serum urea: Creatinine ratio in dogs with babesiosis, haemolytic anaemia, and experimental haemoglobinaemia. Vet J. 2012;191(2):253-256.

34. Malherbe W. Clinico-pathological studies of Babesia canis infection in dogs. The influence of the infection on kidney function. J S Afr Vet Assoc. 1966;37:261-264.

35. Lobetti RG, Reyers F, Nesbit JW. The comparative role of haemoglobinaemia and hypoxia in the development of canine babesial nephropathy. J S Afr Vet Assoc. 1996;67(4):188-198.

36. Lobetti RG, Reyers F. Met-haemoglobinuria in naturally occurring Babesia canis infection. J S Afr Vet Assoc. 1996;67(2):88-90.

37. Jacobson LS. Cerebellar ataxia as a possible complication of babesiosis in two dogs. J S Afr Vet Assoc. 1994;65(3):130-131.

38. Jacobson LS, Lobetti RG. Rhabdomyolysis as a complication of canine babesiosis. J Small Anim Pract. 1996;37(6):286-291. 
39. Dvir E, Lobetti RG, Jacobson LS, Pearson J, Becker PJ. Electrocardiographic changes and cardiac pathology in canine babesiosis. J Vet Cardiol. 2004;6(1):15-23.

40. Conrad P, Thomford J, Yamane I, et al. Hemolytic anemia caused by Babesia gibsoni infection in dogs. J Am Vet Med Assoc. 1991;199(5): 601-605.

41. Farwell GE, LeGrand EK, Cobb CC. Clinical observations on Babesia gibsoni and Babesia canis infections in dogs. J Am Vet Med Assoc. 1982;180(5):507-511.

42. Birkenheuer AJ, Levy MG, Savary KC, Gager RB, Breitschwerdt EB. Babesia gibsoni infections in dogs from North Carolina. J Am Anim Hosp Assoc. 1999;35(2):125-128.

43. Slade DJ, Lees GE, Berridge BR, et al. Resolution of a proteinuric nephropathy associated with Babesia gibsoni infection in a dog. $J \mathrm{Am}$ Anim Hosp Assoc. 2011;47(6):e138-e144.

44. Tasaki Y, Miura N, Iyori K, et al. Generalized alopecia with vasculitislike changes in a dog with babesiosis. J Vet Med Sci. 2013;75(10): 1367-1369.

45. Fraga E, Barreiro JD, Goicoa A, et al. Abdominal ultrasonographic findings in dogs naturally infected with babesiosis. Vet Radiol Ultrasound. 2011;52(3):323-329.

46. Koma LM, Kirberger RM, Leisewitz AL, et al. Comparison of effects of uncomplicated canine babesiosis and canine normovolaemic anaemia on abdominal splanchnic Doppler characteristics - a preliminary investigation. J S Afr Vet Assoc. 2005;76(3):138-145.

47. Sweers L, Kirberger RM, Leisewitz AL, et al. The scintigraphic evaluation of the pulmonary perfusion pattern of dogs hospitalised with babesiosis. J S Afr Vet Assoc. 2008;79(2):76-83.

48. Schetters TP, Kleuskens JA, Van De Crommert J, et al. Systemic inflammatory responses in dogs experimentally infected with Babesia canis; a haematological study. Vet Parasitol. 2009;162(1-2):7-15.

49. Mathe A, Voros K, Nemeth T, et al. Clinicopathological changes and effect of imidocarb therapy in dogs experimentally infected with Babesia canis. Acta Vet Hung. 2006;54(1):19-33.

50. Furlanello T, Fiorio F, Caldin M, Lubas G, Solano-Gallego L. Clinicopathological findings in naturally occurring cases of babesiosis caused by large form Babesia from dogs of northeastern Italy. Vet Parasitol. 2005;134(1-2):77-85.

51. Scheepers E, Leisewitz AL, Thompson PN, Christopher MM. Serial haematology results in transfused and non-transfused dogs naturally infected with Babesia rossi. J S Afr Vet Assoc. 2011;82(3): 136-143.

52. Goddard A, Wiinberg B, Schoeman JP, Kristensen AT, Kjelgaard-Hansen M. Mortality in virulent canine babesiosis is associated with a consumptive coagulopathy. Vet J. 2013;196(2):213-217.

53. Rafaj RB, Matijatko V, Kis I, et al. Alterations in some blood coagulation parameters in naturally occurring cases of canine babesiosis. Acta Vet Hung. 2009;57(2):295-304.

54. Baric Rafaj R, Kules J, Selanec J, et al. Markers of coagulation activation, endothelial stimulation, and inflammation in dogs with babesiosis. J Vet Intern Med. 2013;27(5):1172-1178.

55. Liebenberg C, Goddard A, Wiinberg B, et al. Hemostatic abnormalities in uncomplicated babesiosis (Babesia rossi) in dogs. J Vet Intern Med. 2013;27(1):150-156.

56. Vaughan-Scott T, Jacobsen LS, Lobetti RG, Reyers F. Serum concentrations of tumor necrosis factor in dogs naturally infected with Babesia canis and its relation to severity of disease. Veterinary Immunology and Immunopathology. 2004;104(1):27-41.

57. Jacobson LS, Lobetti RG, Becker P, Reyers F, Vaughan-Scott T. Nitric oxide metabolites in naturally occurring canine babesiosis. Vet Parasitol. 2002;104(1):27-41.

58. Lobetti RG, Mohr AJ, Dippenaar T, Myburgh E. A preliminary study on the serum protein response in canine babesiosis. J S Afr Vet Assoc. 2000;71(1):38-42.

59. Matijatko V, Mrljak V, Kis I, et al. Evidence of an acute phase response in dogs naturally infected with Babesia canis. Vet Parasitol. 2007; 144(3-4):242-250.
60. Koster LS, Van Schoor M, Goddard A, et al. C-reactive protein in canine babesiosis caused by Babesia rossi and its association with outcome. J S Afr Vet Assoc. 2009;80(2):87-91.

61. Suzuki K, Wakabayashi H, Takahashi M, et al. A Possible treatment strategy and clinical factors to estimate the treatment response in Babesia gibsoni infection. J Vet Med Sci. 2007;69(5): $563-568$.

62. Kjelgaard-Hansen M, Goddard A, Wiinberg B, Koster L, Schoeman JP. Monocyte chemoattractant protein-1 concentration is a marker of mortality in canine babesiosis caused by Babesia rossi. J Vet Intern Med. 2010;24(3):706

63. Leisewitz AL, Jacobson LS, de Morais HS, Reyers F. The mixed acid-base disturbances of severe canine babesiosis. J Vet Intern Med. 2001;15(5):445-452.

64. Keller N, Jacobson LS, Nel M, et al. Prevalence and risk factors of hypoglycemia in virulent canine babesiosis. $J$ Vet Intern Med. 2004;18(3):265-270.

65. Nel M, Lobetti RG, Keller N, Thompson PN. Prognostic value of blood lactate, blood glucose, and hematocrit in canine babesiosis. JVet Intern Med. 2004;18(4):471-476.

66. Schoeman JP, Rees P, Herrtage ME. Endocrine predictors of mortality in canine babesiosis caused by Babesia canis rossi. Vet Parasitol. 2007;148(2):75-82.

67. Reyers F. Is the azotaemia in canine babesiosis an indication of renal disease. 9th Faculty Day, University of Pretoria, Faculty of Veterinary Science $1992 ; 17$.

68. Van Zyl M. Prediction of Survival in Hospitalised Cases of Canine Babesiosis [Dissertation]. Pretoria: University of Pretoria, South Africa; 1995.

69. de Scally MP, Lobetti RG, Reyers F, Humphris D. Are urea and creatinine values reliable indicators of azotaemia in canine babesiosis? J S Afr Vet Assoc. 2004;75(3):121-124.

70. Lobetti RG. Cardiac involvement in canine babesiosis. J SAfr Vet Assoc. 2005;76(1):4-8.

71. Jacobson LS, Lobetti RG, Vaughan-Scott T. Blood pressure changes in dogs with babesiosis. J S Afr Vet Assoc. 2000;71(1):14-20.

72. Bohm M, Leisewitz AL, Thompson PN, Schoeman JP. Capillary and venous Babesia canis rossi parasitaemias and their association with outcome of infection and circulatory compromise. Vet Parasitol. 2006;141(1-2):18-29.

73. Lobetti R, Kirberger R, Keller N, Kettner F, Dvir E. NT-ProBNP and cardiac troponin I in virulent canine babesiosis. Vet Parasitol. 2012; 190(3-4):333-339.

74. Lobetti R, Dvir E, Pearson J. Cardiac troponins in canine babesiosis. J Vet Intern Med. 2002;16(1):63-68.

75. Tilley LP. Analysis of canine P-QRS-T deflections. Philadelphia: Lea \& Febiger; 1992.

76. Kock N, Kelly P. Massive hepatic necrosis associated with accidental imidocarb dipropionate toxicosis in a dog. J Comp Pathol. 1991;104(1): $113-116$.

77. Miller DM, Swan GE, Lobetti RG, Jacobson LS. The pharmacokinetics of diminazene aceturate after intramuscular administration in healthy dogs. J S Afr Vet Assoc. 2005;76(3):146-150.

78. Lin MY, Huang HP. Use of a doxycycline-enrofloxacin-metronidazole combination with/without diminazene diaceturate to treat naturally occurring canine babesiosis caused by Babesia gibsoni. Acta Vet Scand. 2010;52:27.

79. Yamasaki M, Harada E, Tamura Y, et al. In vitro and in vivo safety and efficacy studies of amphotericin B on Babesia gibsoni. Vet Parasitol. 2014;205(3-4):424-433.

80. Birkenheuer AJ, Levy MG, Breitschwerdt EB. Efficacy of combined atovaquone and azithromycin for therapy of chronic Babesia gibsoni (Asian genotype) infections in dogs. J Vet Intern Med. 2004; 18(4):494-498.

81. Matsuu A, Koshida Y, Kawahara M, et al. Efficacy of atovaquone against Babesia gibsoni in vivo and in vitro. Vet Parasitol. 2004; 124(1-2):9-18. 
82. Iguchi A, Shiranaga N, Matsuu A, Hikasa Y. Efficacy of Malarone $\left({ }^{\circledR}\right)$ in dogs naturally infected with Babesia gibsoni. J Vet Med Sci. 2014;76(9):1291-1295.

83. Jacobson LS, Swan GE. Supportive treatment of canine babesiosis. J S Afr Vet Assoc. 1995;66(2):95-105.

84. Birkenheur AJ. Canine and Feline Infectious Diseases. St Louis: Elsevier; 2014.

85. Last RD, Hill JM, Matjila PT, Reme CA. A field trial evaluation of the prophylactic efficacy of amitraz-impregnated collars against canine babesiosis (Babesia canis rossi) in South Africa. J S Afr Vet Assoc. 2007;78(2):63-65.

86. Vercammen F, De Deken R, Maes L. Duration of protective immunity in experimental canine babesiosis after homologous and heterologous challenge. Vet Parasitol. 1997;68(1-2):51-55.
87. Moreau Y, Vidor E, Bissuel G, Dubreuil N. Vaccination against canine babesiosis: an overview of field observations. Trans $R$ Soc Trop Med Hyg. 1989;83 Suppl:95-96.

88. Schetters TP, Kleuskens J, Carcy B, Gorenflot A, Vermeulen A. Vaccination against large Babesia species from dogs. Parasitologia. 2007;49 Suppl 1:13-17.

89. Schetters TP, Kleuskens JA, Scholtes NC, Pasman JW, Goovaerts D. Vaccination of dogs against Babesia canis infection. Vet Parasitol. 1997;73(1-2):35-41.

\section{Publish your work in this journal}

Veterinary Medicine: Research and Reports is an international, peer-reviewed, open access journal publishing original research, case reports, editorials, reviews and commentaries on all areas of veterinary medicine. The manuscript management system is completely online and includes a very quick and fair peer-review system.
Visit http://www.dovepress.com/testimonials.php to read real quotes from published authors. 